\section{P-181 THE PREVENTION OF OCCUPATIONAL HEAT STRESS IN SUGARCANE WORKERS IN NICARAGUA: AN INTERPRETATIVE PHENOMENOLOGICAL ANALYSIS}

${ }^{1}$ Felipe Pacheco-Zenteno, Jason Glaser, Ilana Weiss, Kristina Gyllensten, Kristina Jakobbson, Esteban Arias. 'University of Gothenburg, Sweden

\subsection{6/OEM-2021-EPI.223}

Introduction Chronic kidney disease of non-traditional origin (CKDnt) is an ongoing epidemic that has taken the lives of tens of thousands of people in Mesoamerica, also affecting other tropical geographies. Occupational heat stress, which will increase worldwide as climate change persists, has been identified as a primary trigger of inflammation and subsequent kidney injury and reduced renal function. At Nicaragua's largest sugarcane mill, the water, rest, shade (WRS) intervention has proven to reduce the risk of heat stress and kidney injury effectively as assessed by the research and policy NGO La Isla Network and their academic partners. However, discrepancies between intervention design and implementation have been found.

Objective This study explores the perceptions of the WRS intervention in the company from the perspective of positions responsible for the workers' environment and heat stress prevention implementation.

Method A qualitative design was used in the study. Twentyone key informants of low and middle management, field assistants, and two members from La Isla Network took part in the study. Semi-structured interviews were used to collect the data. Interviews' transcriptions were analyzed using interpretative phenomenological analysis.

Results Four main themes were developed in the analysis of the data: 'A worthwhile struggle,' 'Culture of care,' 'Traditional production culture versus Culture of care,' and 'The importance of the formalization of care.' Each theme contained sub-themes, all of which were further discussed in the light of organizational psychology.

Conclusion Discretionary differences resulting in low and middle management prioritizing production over health protection appeared to relate to a fair part of the implementation challenges and indicate that more efforts are needed to align operations' production and health goals. Education enhancement might be necessary, while further focus on health metrics for performance assessment might offer an opportunity to level perceived incentives and value of health and production.

\section{P-183 THE COSTS IMPUTABLE TO WORK STRESS IN EUROPEAN COUNTRIES: HOW MUCH DOES IT COST FROM A SOCIETAL PERSPECTIVE? A STUDY PROTOCOL}

${ }^{1}$ Hélène Sultan-Taïeb, Tania Villeneuve, Jean-François Chastang, Isabelle Niedhammer. ${ }^{1}$ Université du Québec à Montréal (UQAM), Canada

\subsection{6/OEM-2021-EPI.224}

Introduction Work-related psychosocial exposures are highly prevalent in European countries and are associated with various health outcomes, especially mental disorders and cardiovascular diseases (CVD). Evaluations of the economic burden of psychosocial work exposures are however very seldom in the literature.

Objective To estimate the annual costs of depression and CVD attributable to work-related psychosocial exposures in 28 European Union countries (EU28).
Methods This study will follow the top-down cost-of-illness (COI) approach, which estimates the economic burden of health conditions in a population from a societal perspective, starting from the total costs of diseases. We will estimate the fractions of these costs that are attributable to work-related psychosocial exposures (attributable fractions, AFs). AF estimates require data on the prevalence of exposure in the whole population, and on the relative risk of developing the disease when exposed to the risk factor. Relative risk estimates will come from a systematic literature review. Prevalence of exposure estimates will derive from an analysis of the 2015 European Working Conditions Survey data. Work-related psychosocial exposures will include the factors from the job strain model, effort-reward imbalance model, and other models/concepts depending on the availability of data.

Results Our evaluation of costs will include: (1) direct healthcare costs as reimbursed by public healthcare systems: outpatient visit fees to physicians and other health professionals, hospitalisations, emergency room visits and medication, (2) direct healthcare costs as out-of-pocket payments paid by patients, depending on the extent of the public healthcare system in each country, (3) indirect costs due to sickness absences at work, to disability leave, early retirement and premature death, and the cost of presenteeism when available.

Conclusion This study will provide original and relevant insights on a macro-level for policy-makers and stakeholders when defining public health priorities and preventive strategies in European countries regarding work stress prevention.

\section{P-191 JOB SATISFACTION AMONG HEALTH CARE PROFESSIONALS WORKING AT THE SURGERY DEPARTMENTS IN NIŠ, SERBIA}

${ }^{1}$ Maja Nikolic, Sonja Novak, Katarina Nikolic. ${ }^{1}$ Faculty of medicine University of Nis, Serbia

\subsection{6/OEM-2021-EPI.225}

Introduction Job satisfaction in health care is an important factor determining quality and performance of a health care system.

Objectives The objectives of this study are to assess job satisfaction among Serbian health care professionals of the surgery clinics and to evaluate factors that could influence the job satisfaction rating.

Methods A cross-sectional study using anonymous self-report questionnaire (Spector, 1994) was performed between June 2016 and March 2017. The study included 158 health care professionals, 33 males (20.9\%) and 125 females (79.1\%), from the surgery departments of Niš, Serbia. The questionnaire with 36 item has nine facet scale (Pay, Promotion, Supervision, Fringe Benefits, Contingent Rewards, Operating Procedures (required rules and procedures), Coworkers, Nature of Work, and Communication) and each facet is assessed with four items, and a total score is computed from all items. Data were analyzed using SPSS version 20.

Results Highest-rated factors of job satisfaction were nature of work (the average score was $15.78 \pm 5.38$, maximum 24) and supervision $(14.90 \pm 5.79)$. Highest-rated stressors were fringe benefits $(10.90 \pm 4.36)$, and promotion (11.26 \pm 4.33). Compared to nurses, a higher total job satisfaction was found among doctors $(p=0.020)$. There was a statistically 